\title{
La réflectivité des neutrons
}

\author{
A. Menelle
}

Laboratoire Léon Brillouin, CEA-Saclay, 91191 Gif-sur-Yvette cedex, France

\section{LES PRINCIPES DE BASE}

I.1. CALCUL DE L'INDICE POUR LES NEUTRONS

I.2. RÉFLEXION SUR UNE SUCCESSION DE COUCHES

I.3. L'INTERFACE IDÉALE ET LA COURBE DE FRESNEL

I.4. CAS DE LA COUCHE HOMOGÈNE SUR UN SUBSTRAT

I.5. LA RUGOSITÉ

\section{LES RÉFLECTOMÈTRES NEUTRONS}

\section{II.1. MESURE D'UNE COURBE DE RÉFLECTIVITÉ EN TEMPS DE VOL \\ II.2. LE RÉFLECTOMÈTRE EROS DU LLB}

\section{MESURES TYPIQUES NEUTRONS}

III.1. SYSTĖMES MÉTALLIQUES

III.2. INTERDIFFUSION ENTRE POLYMERES

III.3. POLYMĖRES EN SURFACE

III.4. INTERFACES SOLIDE LIQUIDES

III.5. UN PEU DE NON SPÉCULAIRE

\section{I.Les principes de base}

Un neutron pouvant être décrit en utilisant un formalisme ondulatoire, la réflectivité de neutrons ${ }^{1}$ est très proche de la réflectivité de rayons $\mathrm{X}$. Le formalisme qui s'applique est le même comme nous le verrons plus loin. Les spécificités sont décrites brièvement ici.

L'interaction neutron matière a lieu directement entre le neutron et le noyau atomique, et non le nuage électronique pour les rayons $X$. Elle est de très courte portée et est généralement représentée par un pseudo-potentiel de Fermi ponctuel et centré sur l'atome. En conséquence, cette interaction dépendra non seulement de l'élément du tableau périodique sondé comme pour les rayons $\mathrm{X}$, mais aussi du type d'atome (différente d'un isotope à l'autre). Cette propriété est énormément utilisée pour l'étude des colloïdes et polymères où l'on remplace souvent l'hydrogène par le deutérium pour marquer les molécules.

L'amplitude de cette interaction neutron matière est tabulée et varie aléatoirement d'un atome à l'autre. Elle peut être répulsive et attractive, et donc caractérisée par un chiffre positif ou négatif. Ceci est différent des rayons $\mathrm{X}$ où elle dépend du nombre d'électrons qui est toujours positif.

L'absorption des neutrons par la matière est faible, et est dans la plupart des cas négligée en réflectivité (sauf pour le bore, le gadolinium, le lithium, ...). Ceci se traduit par un indice sans partie imaginaire.

Le neutron a un spin $\pm 1 / 2$ qui interagit avec les atomes porteur de moment magnétique. Ceci permettra de mesurer la distribution des moments magnétiques dans les couches minces 


\section{I.Calcul de l'indice pour les neutrons}

On définit un faisceau de neutron se réfléchissant sur une surface plane avec un angle d'incidence $\theta$ (voir Figure.1). Cette surface est définie par l'interface entre le vide $(n=1)$ et un milieu d'indice de réfraction $\mathrm{n}$. Pour une longueur d'onde $\lambda$, le vecteur de diffusion incident est définit par :

$\mathrm{k}=\frac{2 \pi}{\lambda}$

Le vecteur de diffusion dans le milieu d'indice $n$ est définit par :

$\mathrm{k}_{\mathrm{n}}^{2}=\mathrm{k}^{2}-4 \pi \mathrm{Nb}$

$\mathrm{N}$ est le nombre d'atomes par unité de volume, et $\mathrm{b}$ est la longueur de diffusion cohérente caractéristique du matériaux formant le milieu d'indice $\mathrm{n}$. Le produit $\mathrm{Nb}$ est appelé densité de longueur de diffusion cohérente. Dans un milieu homogène, l'indice de réfraction $\mathbf{n}$ est défini comme le rapport des vecteur d'onde dans le matériaux et dans le vide $:^{2}$ :

$\mathrm{n}=\frac{\mathrm{k}_{\mathrm{n}}}{\mathrm{k}}=1-\frac{\lambda^{2}}{2 \pi} \mathrm{Nb}$

On retrouve l'écriture de l'indice pour les rayons $\mathrm{X}$ si on néglige la partie imaginaire, et que l'on remplace $\mathrm{Nb}$ par le produit de la densité électronique par le rayon classique de l'électron.

A l'interface entre le vide et le milieu d'indice $\mathrm{n}$, on écrit la loi de réfraction de Descartes'law

$\cos \theta=\mathrm{n} \cos \theta_{\mathrm{n}}$

Dans ce cas, il y a réflexion totale si $\theta \leq \theta_{\mathrm{c}}$, où $\theta_{\mathrm{c}}$ est défini de façon à avoir $\theta_{\mathrm{n}}=0$.

Ceci conduit à :

$\cos \theta_{\mathrm{c}}=\mathrm{n}$

$\mathrm{n}$ étant proche de 1 on obtient en développant :

$\mathrm{n}^{2} \approx 1-\frac{\lambda^{2}}{\pi} \mathrm{Nb}$

De (5) et (6), on tire :

$1-\left(\sin \theta_{c}\right)^{2}=1-\frac{\lambda^{2}}{\pi} \mathrm{Nb}$

ce qui est équivalent à :

$\sin \theta_{\mathrm{c}}=\sqrt{\frac{\mathrm{Nb}}{\pi}} \lambda=\alpha \lambda$

Les paramètre $\alpha$ ou $\mathrm{Nb}$ sont utilisés pour caractériser le matériaux.

Le Tableau 1 donne quelques valeurs typiques de densité de longueur de diffusion ${ }^{3}$.

Tableau 1 : Densité de longueur de diffusion de quelques matériaux. $\mathrm{N}_{\mathrm{A}}$ est le nombre d'Avogadro.

\begin{tabular}{ccccc}
\hline \hline & $\mathrm{Ni}$ & $\mathrm{Ti}$ & $\mathrm{Si}$ & $\mathrm{SiO}_{2}$ \\
\hline density d $\left(\mathrm{g} \cdot \mathrm{cm}^{-3}\right)$ & 8,9 & 4,51 & 2,33 & 2,5 \\
Atomic Mass $\mathrm{M}\left(\mathrm{g}^{\left.-\mathrm{mol}^{-1}\right)}\right.$ & 58,71 & 47,9 & 28,09 & 60,09 \\
$\mathrm{~N}=\mathrm{dN}_{\mathrm{A}} \mathrm{M}^{-1}\left(\mathrm{~cm}^{-3}\right)$ & $9,13 \times 10^{22}$ & $5,67 \times 10^{22}$ & $5,00 \times 10^{22}$ & $2,51 \times 10^{22}$ \\
$\mathrm{~b}\left(\mathrm{~cm}^{-2}\right)$ & $1,03 \times 10^{-12}$ & $-0,3438 \times 10^{-12}$ & $0,41491 \times 10^{-12}$ & $1,58 \times 10^{-12}$ \\
$\mathbf{N b}\left(\AA^{-2}\right)$ & $\mathbf{9 , 4 \times 1 0 ^ { - 6 }}$ & $\mathbf{- 1 , 9 5 \times 1 0 ^ { - 6 }}$ & $\mathbf{2 , 0 7 \times 1 0 ^ { - 6 }}$ & $\mathbf{3 , 9 6 \times 1 0 ^ { - 6 }}$ \\
\hline \hline
\end{tabular}




\section{Remarque:}

Contrairement aux rayons $X$ où les indices sont toujours plus petits que 1 , certains produits peuvent présenter des indices supérieurs à 1 pour les neutrons. Ceci se traduit par l'absence de plateau de réflexion totale pour le titane et l'eau en particulier.

\subsection{Réflexion sur une succession de couches}

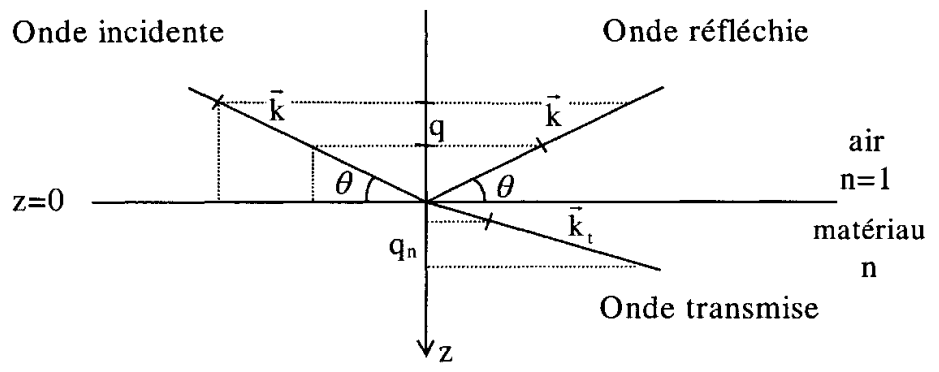

Figure.1 : Réflexion sur une surface plane.

La projection du vecteur d'onde sur l'axe z (perpendiculaire à la surface) défini la variable $\mathrm{q}$ :

$$
q=\frac{2 \pi}{\lambda} \sin \theta
$$

Si le système étudié est composé de nombreuses couches, chacune ayant pour indice $\mathrm{n}_{\mathrm{p}}$, la propagation de l'onde plane dans la couche $\mathrm{p}$ et la couche $\mathrm{p}+1$ peut s'écrire de la manière suivante :

$$
\begin{aligned}
& \psi_{z}\left(z_{p}\right)=\mathrm{A}_{p} \exp \left(\mathrm{iq}_{p} \mathrm{z}_{p}\right)+\mathrm{B}_{p} \exp \left(-\mathrm{iq}_{p} \mathrm{z}_{p}\right) \\
& \psi_{z}\left(z_{p+1}\right)=\mathrm{A}_{p+1} \exp \left(\mathrm{iq}_{p+l} \mathrm{z}_{p+1}\right)+\mathrm{B}_{p+1} \exp \left(-\mathrm{iq}_{p+1} \mathrm{z}_{p+1}\right)
\end{aligned}
$$

où $\mathrm{i}^{2}=-1$, et $\mathrm{A}_{p}$ et $\mathrm{B}_{p}$ sont respectivement les amplitudes de l'onde se propageant vers l'intérieur et l'extérieur du matériau. Nous avons également :

$$
\begin{aligned}
& \mathrm{q}_{p}^{2}=\mathrm{q}^{2}-4 \pi \mathrm{Nb}_{p} \\
& \mathrm{q}_{p+1}^{2}=\mathrm{q}^{2}-4 \pi \mathrm{Nb}_{p+1}
\end{aligned}
$$

Les conditions de continuité de l'onde et de sa dérivée à l'interface $\mathrm{p} / \mathrm{p}+1$ s'écrivent :

$$
\begin{aligned}
& \Psi_{z}\left(z_{p}\right)=\Psi_{z}\left(z_{p+1}\right)=u\left(z_{p / p+1}\right) \\
& \Psi_{z}^{\prime}\left(z_{p}\right)=\Psi_{z}^{\prime}\left(z_{p+1}\right)=u^{\prime}\left(z_{p / p+1}\right)
\end{aligned}
$$

La réflectivité en $z_{p / p+1}$ est définie comme le rapport de l'intensité du faisceau réfléchi par la couche $\mathrm{p}+1$ sur l'intensité incidente sur cette même couche. Elle s'écrit :

$$
\mathrm{R}=\frac{\left|\mathrm{B}_{p}\right|^{2}}{\left|\mathrm{~A}_{p}\right|^{2}}=\left|\frac{1-\frac{\mathrm{u}^{\prime}\left(\mathrm{z}_{p / p+1}\right)}{\mathrm{iq}_{p} \mathrm{u}^{2}\left(\mathrm{z}_{p / p+1}\right)}}{1+\frac{\mathrm{u}^{\prime}\left(\mathrm{z}_{p / p+1}\right)}{\mathrm{iq}_{p} \mathrm{u}\left(\mathrm{z}_{p / p+l}\right)}}\right|^{2}
$$

où $u\left(z_{p / p+1}\right)$ et $u^{\prime}\left(z_{p / p+1}\right)$ sont des fonctions de $z_{p / p+1}$ et $q_{p+1}$. 
A partir de cette équation il est possible de calculer la réflectivité pour la dernière interface (dernière couche/matériaux massif), puis calculer récursivement la réflectivité à chaque interface jusqu'à la surface, et ainsi obtenir la réflectivité du système étudié.

\section{L.3.Linterface idéale et la courbe de Fresnel}

Les milieux $\mathrm{p}$ et $\mathrm{p}+\mathrm{l}$ sont remplacés par le vide et un substrat d'indice $\mathrm{n}$. Si l'interface est parfaite sans rugosité, un tel système est un dioptre, et sa réflectivité, la réflectivité de Fresnel $\left(\mathrm{R}_{\mathrm{F}}\right)$. Dans ce cas, dans le matériau, $\mathrm{B}_{p+l}=\mathrm{B}_{\mathrm{s}}=0$ pour l'équation (10) car aucune intensité ne revient de $\mathrm{z}=\infty$.

On peut écrire la réflectivité de Fresnel :

$R_{F}=\left|\frac{B}{A}\right|^{2}=\left|\frac{q-q_{s}}{q+q_{s}}\right|^{2}$

ou :

$\mathrm{R}_{\mathrm{F}}=\frac{1-\left(1-\left(\frac{\mathrm{q}_{\mathrm{c}}}{\mathrm{q}}\right)^{2}\right)^{1 / 2}}{1+\left(1-\left(\frac{\mathrm{q}_{\mathrm{c}}}{\mathrm{q}}\right)^{2}\right)^{1 / 2}}$

oùe $\mathrm{q}_{\mathrm{c}}$ et $\mathrm{q}_{\mathrm{s}}$ sont obtenu par l'équation (11) :

$\mathrm{q}_{\mathrm{c}}^{2}=4 \pi \mathrm{Nb}$

$\mathrm{q}_{\mathrm{s}}^{2}=\mathrm{q}^{2}-4 \pi \mathrm{Nb}_{\mathrm{s}}=\mathrm{q}^{2}-\mathrm{q}_{\mathrm{c}}^{2}$

Une courbe de réflectivité de Fresnel est représentée sur la Figure 2

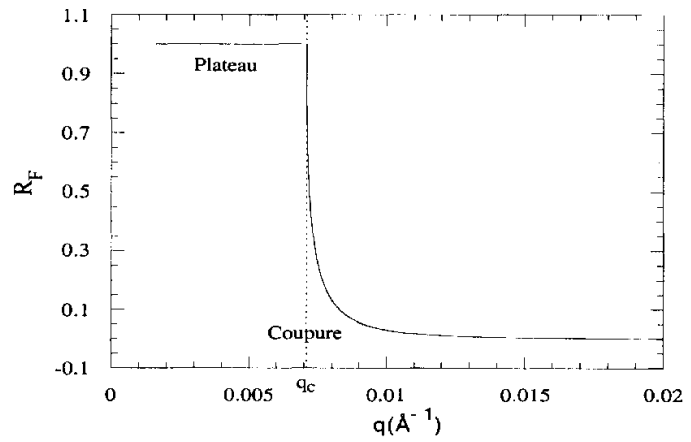

Figure 2 : Représentation de $R_{F}$ en fonction de $q$. (calculé pour $N_{s}=4 \times 10^{-6} \AA^{-2}$ ) 


\subsection{Cas de la couche homogène sur un substrat}

On s'intéresse au système représenté sur la

Figure 3. Les trois milieux sont : l'air $(n=1)$, une couche homogène d'indice $n_{1}$ d'épaisseur $d$ et de densité de longueur de diffusion $\mathrm{Nb}_{1}$, et le substrat d'indice $\mathrm{n}_{\mathrm{s}}$ de densité de longueur de diffusion $\mathrm{Nb}_{\mathrm{s}}$ et d'épaisseur infinie. L'interface air/couche est en $\mathrm{z}=0$, l'interface couche/substrat est en $\mathrm{z}=\mathrm{d}$.

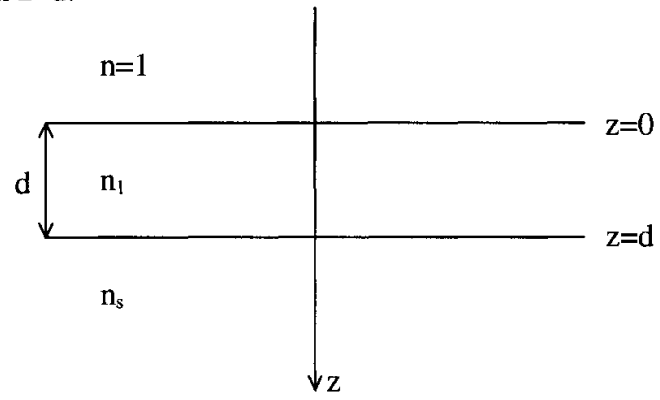

Figure 3 : Couche homogène sur substrat.

Après quelques calculs, on obtient :

$$
\mathrm{R}=\frac{\cos \left(2 \mathrm{q}_{1} \mathrm{~d}\right)\left[1+\left(\frac{\mathrm{q}_{\mathrm{s}}}{\mathrm{q}}\right)^{2}-\left(\frac{\mathrm{q}_{1}}{\mathrm{q}}\right)^{2}-\left(\frac{\mathrm{q}_{\mathrm{s}}}{\mathrm{q}_{1}}\right)^{2}\right]+1-4 \frac{\mathrm{q}_{\mathrm{s}}}{\mathrm{q}}+\left(\frac{\mathrm{q}_{\mathrm{s}}}{\mathrm{q}}\right)^{2}+\left(\frac{\mathrm{q}_{\mathrm{l}}}{\mathrm{q}}\right)^{2}+\left(\frac{\mathrm{q}_{\mathrm{s}}}{\mathrm{q}_{1}}\right)^{2}}{\cos \left(2 \mathrm{q}_{1} \mathrm{~d}\right)\left[1+\left(\frac{\mathrm{q}_{\mathrm{s}}}{\mathrm{q}}\right)^{2}-\left(\frac{\mathrm{q}_{1}}{\mathrm{q}}\right)^{2}-\left(\frac{\mathrm{q}_{\mathrm{s}}}{\mathrm{q}_{\mathrm{l}}}\right)^{2}\right]+1+4 \frac{\mathrm{q}_{\mathrm{s}}}{\mathrm{q}}+\left(\frac{\mathrm{q}_{\mathrm{s}}}{\mathrm{q}}\right)^{2}+\left(\frac{\mathrm{q}_{1}}{\mathrm{q}}\right)^{2}+\left(\frac{\mathrm{q}_{\mathrm{s}}}{\mathrm{q}_{\mathrm{l}}}\right)^{2}}
$$

On peut voir sur cette équation que la courbe de $\mathrm{R}$ en fonction de q présentera des oscillations dont la fréquence sera donnée par $2 \mathrm{q}_{1} \mathrm{~d}=\mathrm{m} \times 2 \pi$. C'est la relation de Bragg corrigée de l'angle critique qui donne : $2 \mathrm{~d} \sqrt{\sin ^{2} \theta_{\mathrm{m}}-\sin ^{2} \theta_{\mathrm{c}}}=\mathrm{m} \lambda$ ( $\mathrm{m}$ is an integer). Ces oscillations sont les franges de Kiessig.

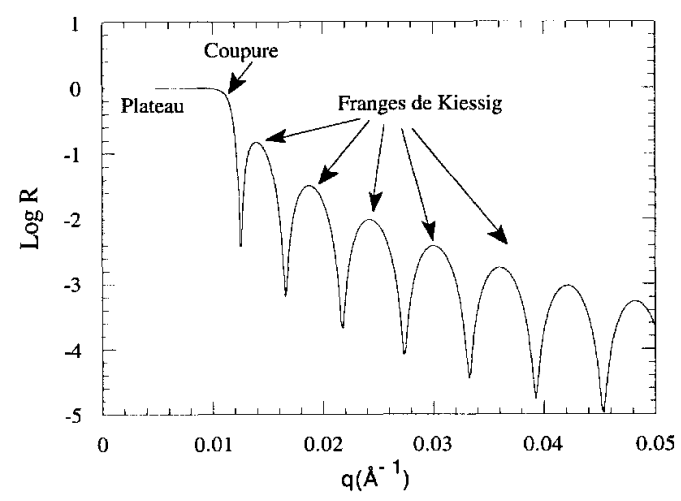

Figure.4 : Réflectivité R calculée pour une couche de $500 \AA ̊$ de nickel sur du silicium. 


\section{L.5.La rugosité}

Un système est décrit par un profil de densité de longueur de diffusion, fonction de la distance à la surface $\mathrm{z}: \mathrm{Nb}(\mathrm{z})$. Jusqu'à présent, nous avons toujours supposé que l'interface entre la couche $\mathrm{P}$ et la couche $\mathrm{p}+1$ était parfaite et présentait une discontinuité de $(\mathrm{Nb})_{p}$ à $(\mathrm{Nb})_{p+1}$ (fonction en escalier). Dans la pratique, les interfaces ne sont pas si abruptes du fait de deux phénomènes différents :

- la rugosité : d'un point à l'autre de la surface, le passage d'une couche à l'autre ne se fait pas toujours à la même distance $\mathrm{z}$ de la surface.

- l'interdiffusion : deux matériaux formant deux couches successives diffusent souvent légèrement l'un dans l'autre créant une transition non abrupte..

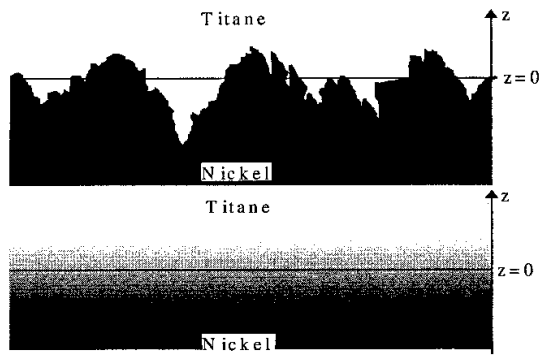

La mesure de la réflectivité spéculaire ne permet pas de distinguer entre la rugosité et l'interdiffusion si la taille des imperfections est plus petite que la longueur de cohérence de l'onde associée au neutron (qui est de l'ordre de quelques microns). Seule la mesure de la diffusion non-spéculaire, absente en cas d'interdiffusion, permet de différentier ces rugosités. Les deux types d'interfaces seront simulées par la même fonction erreur :

$\operatorname{erf}\left(\frac{\mathrm{z}-\mathrm{z}_{p / p+1}}{\sigma_{p / p+1}}\right)=\frac{2}{\sqrt{\pi}} \int_{0}^{\left(\mathrm{z}-\mathrm{z}_{p / p+1}\right) / \sigma_{p / p+1}} \mathrm{e}^{-\mathrm{t}^{2}} \mathrm{dt}$

où $p / p+l$ est l'interface entre deux couches successives. La courbe représentant cette fonction erreur présente un point d'inflexion en $\mathrm{z}_{p / p+1}, \sigma_{p / p+1}$ est l'inverse de la pente à la tangente à la courbe en $\mathrm{z}_{p / p+1}$. L'épaisseur de l'interface est donnée par $2 \sigma_{p / p+1}$.

On peut montrer que l'introduction de l'équation (18) dans le calcul de la réflectivité conduit à multiplier la réflectivité $\mathrm{R}$ obtenue dans le cas d'une interface parfaite entre $\mathrm{p}$ et $\mathrm{p}+1$, par un facteur type Debye-Waller factor ayant pour valeur :

$\mathrm{DW}=\exp \left(-4 \mathrm{q}_{p} \mathrm{q}_{p+1} \sigma_{p / p+1}^{2}\right)$

avec $\mathrm{q}_{p}$ et $\mathrm{q}_{p+l}$ donnés par l'équation (11).

Une autre méthode ${ }^{4}$ également utilisée consiste à discrétiser le profil de densité en un grand nombre de couches présentant une faible variation de $\mathrm{Nb}$ de l'une à l'autre. Toutefois, dans ce cas, le temps de calcul devient plus long.

La Figure.5 montre l'effet de la rugosité sur une courbe de réflectivité calculée pour une couche de nickel déposée sur un substrat de silicium. 


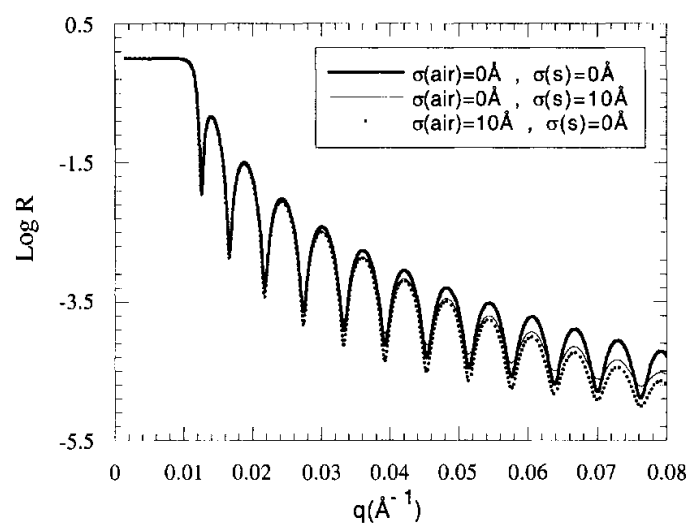

Figure.5 : Effet de la rugosité sur la courbe de réflectivité calculée pour une couche de $500 \AA$ de nickel déposée sur un substrat de silicium. Ligne épaisse : $\sigma_{\mathrm{s}}=\sigma_{\mathrm{arr}}=0 \AA$; ligne fine: $\sigma_{\mathrm{s}}>0$; points : $\sigma_{\mathrm{air}}>0$.

\section{II.Les réflectomètres neutrons}

Les réflectomètres neutrons peuvent fonctionner de deux manières distinctes. La plus simple, similaire aux réflectomètres rayons $\mathrm{X}$ de laboratoire, utilise une longueur d'onde fixe comprise entre $1.5 \AA$ et $6 \AA$. Dans ce cas, un pinceau fin de neutrons est envoyé sur l'échantillon sous une incidence rasante, et on mesure le faisceau réfléchi, pas à pas, en tournant l'échantillon d'un angle $\theta$ et le détecteur d'un angle $2 \theta$. Du fait du faible flux de neutrons, seule la taille de ces appareils les différencie notablement des appareils à rayons $\mathrm{X}$ de laboratoire.

Toutefois les neutrons permettent l'utilisation d'une autre méthode dite de temps de vol pour mesurer des courbes de réflectivité. C'est un peu l'équivalent de la méthode par dispersion d'énergie pour les rayons $\mathrm{X}$. Elle est décrite ici.

\section{Џ.1.Mesure d'une courbe de réflectivité en temps de vol}

La mesure du coefficient de réflexion sur une surface est simple : on divise l'intensité mesurée dans le faisceau réfléchie par celle mesurée dans le faisceau incident, et ceci pour chaque valeur de $\lambda$, et donc de $\mathrm{q}=\frac{2 \pi \sin \theta}{\lambda}$. Une des méthodes employée pour décrire cette courbe est de travailler à angle $\theta$ fixé, et faire varier la longueur d'onde $\lambda$. Le faisceau incident contient toutes les longueurs d'onde. La vitesse du neutron dépendant fortement de sa longueur d'onde, on obtient celle ci en mesurant le temps mis pour atteindre le compteur. C'est le principe de la méthode du temps de vol $^{5,6}$.

Cette technique permet de séparer les neutrons de différentes longueur d'onde assemblés dans une même impulsion brève. Le temps $\mathrm{t}$ nécessaire à un neutron de longueur d'onde $\lambda$ pour parcourir la distance $\mathrm{L}$ est donné par : 
$\lambda=\frac{\mathrm{h}}{\mathrm{mL}} \mathrm{t}$

où $\mathrm{h}$ est la constante de Plank et $\mathrm{m}$ la masse du neutron. Ceci est équivalent à :

$\lambda(\AA)=\frac{\mathrm{t}(\mathrm{s})}{252,7 \mathrm{~L}(\mathrm{~m})}$

Un hacher (chopper) est utilisé pour fabriquer l'impulsion de neutrons. Chaque impulsion est formée au temps $t=0$. La mesure du temps $t$ mis par le neutron pour atteindre le détecteur situé à la distance $\mathrm{L}$ du hacheur donne la longueur d'onde. La vitesse de rotation du hacheur est choisi de façon à ce que les neutrons les plus lents d'une impulsion arrivent sur le détecteur avant les neutrons les plus rapides de la suivante.

\section{II.2.Le réflectomètre EROS du LLB}

Les neutrons produits par le réacteur Orphée sont thermalisés par une source froide à hydrogène liquide $(\mathrm{T}=20 \mathrm{~K})$. Un guide de neutrons les amène jusqu'au réflectomètre situé à environ $30 \mathrm{~m}$ de la source. Là une faisceau dit "blanc", contenant toutes les longueurs d'ondes, est disponible. La gamme réellement utilisable va de 2 à $25 \AA$. Un schéma du réflectomètre est donné sur la Figure 6 . Le hacheur est constitué d'un disque de $40 \mathrm{~cm}$ de diamètre absorbant les neutrons. Une fenêtre de taille ajustable de largeur $25 \mathrm{~mm}$ et de hauteur comprise entre 5 et $40 \mathrm{~mm}$ est située à la périphérie du disque. La résolution peut ainsi être facilement adaptée à l'échantillon étudié. Le faisceau parcoure ensuite un long collimateur sous vide $(4 \mathrm{~m})$. La largeur des fentes d'entrée det de sortie de ce collimateur peuvent être ajustées entre 0.5 et $5 \mathrm{~mm}$. Le faisceau incident est ensuite réfléchi par l'échantillon incliné d'un $\theta$. L'angle maximum utilisable est de $5^{\circ}$, ce qui permet de couvrir un domaine de vecteur de diffusion $q$ de 0.004 à $0.1 \AA^{-1}$. La plupart des expériences sont réalisées en utilisant un seul angle de mesure; toutefois, parfois deux sont nécessaires $\left(1^{\circ}\right.$ et $4^{\circ}$ par exemple). Le faisceau réfléchi est mesuré à l'aide d'un détecteur à ${ }^{3} \mathrm{He}$. La réflectivité minimale qui peut être mesurée est de l'ordre de $10^{-6}$.

La position verticale du détecteur doit être ajustée. $S i h_{0}$ et $h_{d}$ sont les positions respectives du faisceau direct et du détecteur à la distance $\mathrm{L}$ du hacheur, l'angle $\theta$ de mesure est donné par :

$\tan (2 \theta)=\frac{h_{d}-h_{0}}{D_{s-d}}$

où $\mathrm{D}_{\mathrm{s}-\mathrm{d}}$ est la distance de l'échantillon au détecteur. 


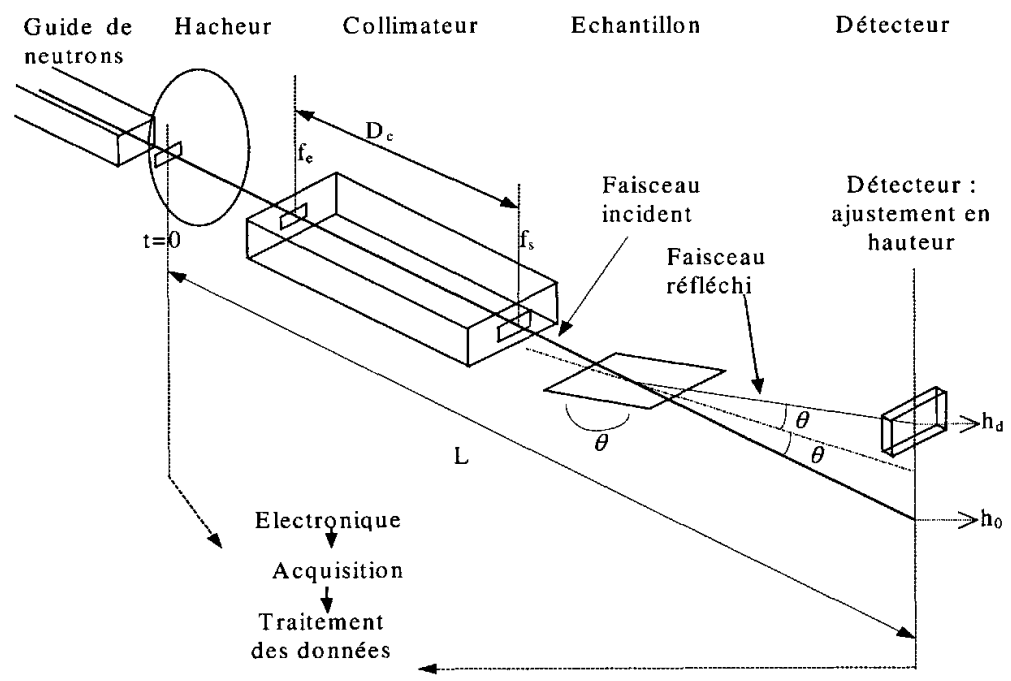

Figure 6 : Schéma du réflectomètre EROS

Ce principe de fonctionnement est utilisé sur tous les réflectomètres installés sur les sources pulsées. Il est également utilisé sur le réflectomètre D17 installé à l'ILL à Grenoble.

\section{III.Mesures typiques neutrons}

Je donne ici quelques exemples de mesures qui permettent de voir les différents type de sujets qui sont étudiés en réflectivité de neutrons (hormis le magnétisme). Ils font tous appel à une ou plusieurs spécificité des neutrons.

\section{III.1.Systèmes métalliques}

Ces 15 dernières années des progrès significatifs ont vu le jour pour la réalisation de dépôts en couches minces d'épaisseur très faible (jusqu'à $50 \AA$ ), très reproductibles et sur de grandes surfaces. Ces développements industriels ont été mis à profit pour la réalisation de dispositifs d'optique neutronique à base de multicouches apériodiques $\mathrm{Ni} / \mathrm{Ti}$ : les supermiroirs. Ces dispositifs permettent d'augmenter artificiellement (par interférences) la longueur du plateau de réflexion total (voir Figure 7). Lorsqu'ils sont utilisés dans la fabrication de guides de neutrons, ils permettent d'augmenter la divergence transmise par le guide d'un facteur proche de 2 et donc le flux d'un facteur voisin de 4. 


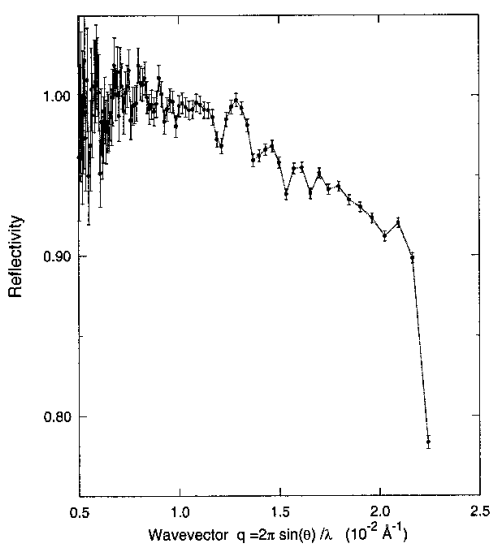

Figure 7 : Courbe de réflectivité caractéristique d'un supermiroir à $2 \theta_{c}$;
La qualité de tels dispositifs dépend essentiellement de la valeur de la réflectivité obtenue sur l'extension du plateau de réflexion totale qui doit être supérieure à $90 \%$. Afin de pouvoir réaliser rapidement de telles mesures sur les grandes quantités de dalles nécessaires à la réalisation d'un guide de neutron, nous avons développer une méthode de mesure spécifique par multiréflexion.

\section{III.2.Interdiffusion entre polymeres}

Les distances facilement accessibles par réflectivité de neutrons sont de l'ordre de $50 \AA$ à $1000 \AA$. Ce sont des distances caractéristiques des systèmes polymèriques. De plus, l'utilisation de la deutération sélective de certains polymère permet de marquer très efficacement les couches à étudier. Par centrifugation (spin coating), on arrive facilement à préparer des couches présentant des rugosités de surface de l'ordre de $10 \AA$. Si l'on met plusieurs de ces couches l'une sur les autres, dès que la température dépasse la température de transition vitreuse, elle vont commencer à interdiffuser l'une dans l'autre. Si l'une de ces couches est deutérée, ceci va se traduire par une rugité plus grande que l'on peut mesurer et qui sera représentative de la longueur d'interdiffussion (voir Figure 8).

Interface amorphe / amorphe

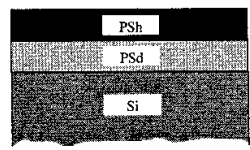

Interface amorphe / élastomère
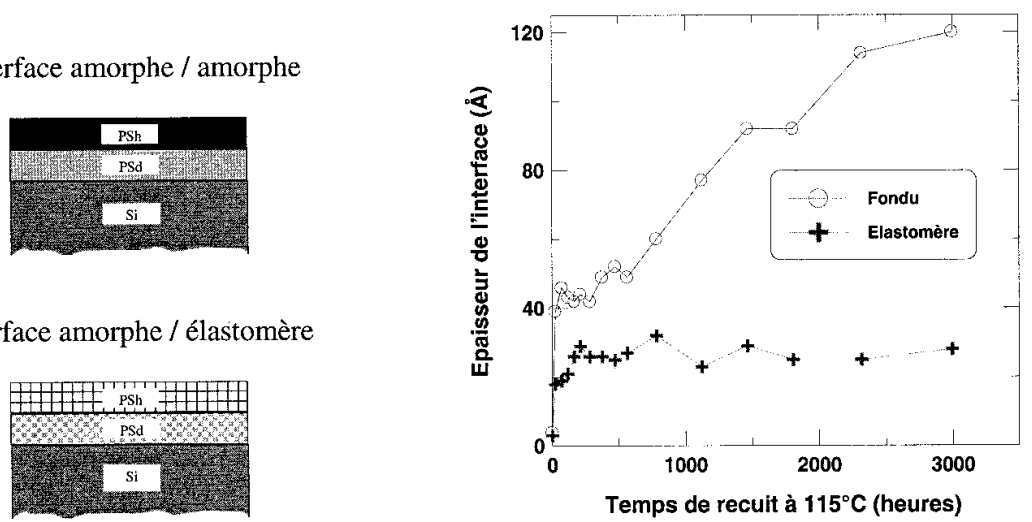

Figure 8 : Evolution de l'épaisseur de l'interface mesurée sur l'échantillon réticulé et non réticulé représenté sur la gauche. 


\section{II.3.polymères en surface}

Les systèmes polymèriques en solution présentent d'intéressantes variations de leurs propriétés pour de légers changement d'environnement comme la température ou la concentration de petites molécules de surfactant. Ils sont en particulier de plus en plus utilisés pour les stabilisations de systèmes diphasiques. Au LLB, il a été étudié, par réflectivité de neutrons, le comportement au voisinage de l'interface eau/air, du poly(N-isopropylacrylamide) (PNIPAM), un polymère soluble dans l'eau jusqu'à une température Tc de $33^{\circ} \mathrm{C}$, proche de la température usuelle du corps humain. Il a été montré ${ }^{7}$ que lorsque l'on élève la température jusqu'à Tc, le PNIPAM forme une couche de plus en plus dense à la surface de l'eau. Par ailleurs, le PNIPAM interagit fortement avec un surfactant anionique, le dodécyl sulfate de sodium (SDS) ce qui permet d'en faire varier ces propriété. Il a pu être montré que l'addition de SDS à une solution de PNIPAM conduisait tout d'abord à une diminution de la concentration de PNIPAM en surface par la formation de micelles (voir Figure 9), mais aussi à une augmentation de la température de transition Tc. Ces résultats prouvent que le couple PNIPAM/SDS présente, pour des applications potentielles, une flexibilité d'utilisation beaucoup plus grande que le polymère utilisé seul.

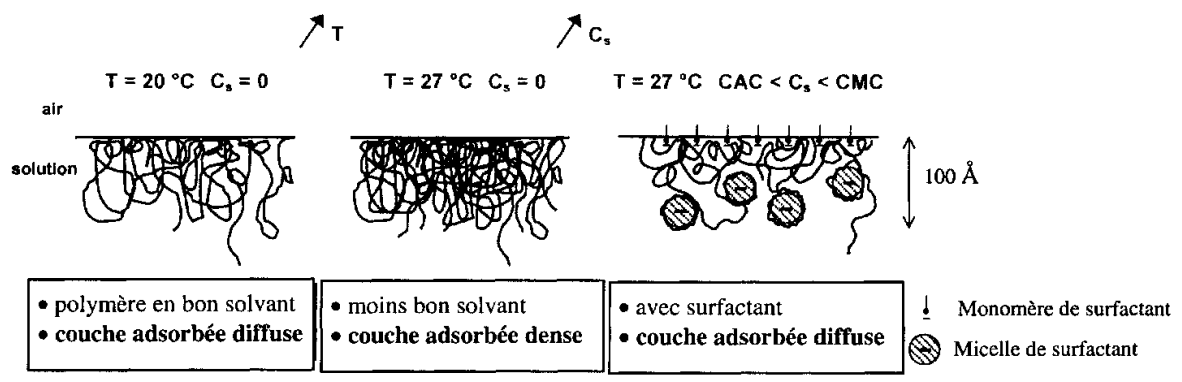

Figure 9 : Structure de la couche de PNIPAM à la surface de l'eau : effet de la température et de la concentration en SDS

\section{III.4.Interfaces solide liquides}

$\mathrm{Du}$ fait de la très faible absorption des neutrons par certains matériaux, on peut aisément étudier les interface solides liquides en traversant soit le liquide soit le solide (voir Figure 11). Le silicium étant particulièrement transparent, nous avons réalisés des mesures d'interdigitation entre des polymères gréffés à la surface (couches de PDMS) et mises en contact avec des fondus de PDMS. Ces couches ont été analysées par réflectivité de neutrons en utilisant une couche greffée deutérée. L'utilisation d'une procédure d'ajustement des courbes de réflectivité de type "maximum d'entropie"8 a permis d'obtenir les profils de concentration des couches greffées sans la supposition "a priori" d'un modèle de profil (voir Figure 10). Les expériences donnent des profils paraboliques correspondants aux prédictions théoriques $^{9-10}$. Leur extension est toutefois légèrement plus faible que celui prévu. 


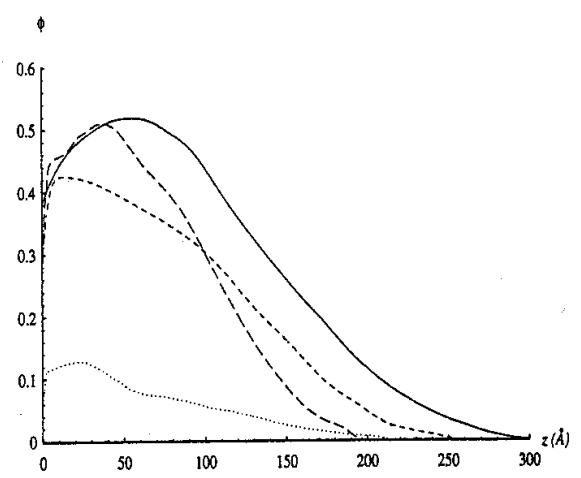

Figure 10 : Profils de couches greffées de PDMSd d'épaisseur $h_{0}$ face à des fondus de PDMSh $\begin{aligned} \ldots \ldots \mathrm{h}_{0} & =30 \AA, \mathrm{mP}=92000 \mathrm{~g} / \mathrm{mole} \\ \mathrm{h}_{0} & =96 \AA, \mathrm{mP} 18000 \mathrm{~g} / \mathrm{mole} \\ -\mathrm{h}_{0} & =65 \AA, \mathrm{mP}=36000 \mathrm{~g} / \mathrm{mole} \\ -\cdots \mathrm{h}_{0} & =69 \AA, \mathrm{mP}=92000 \mathrm{~g} / \mathrm{mole}\end{aligned}$

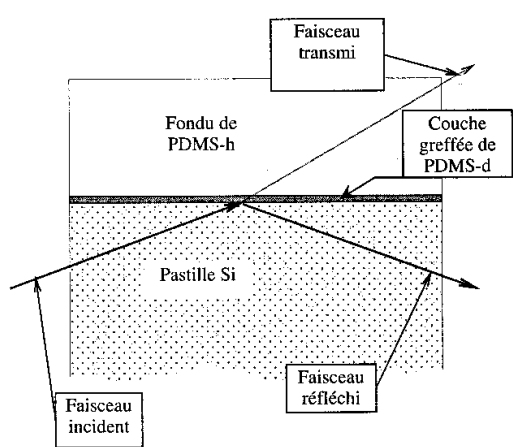

Figure 11 : Schéma du montage utilisé

\section{III.5.Un peu de non spéculaire}

Les mesures de diffusion de neutrons non spéculaire peuvent être faites comme en rayons X. Elles peuvent être réalisées soit en utilisant un spectromètre à longueur d'onde fixe, soit un spectromètre fonctionnant en temps de vol. Dans tous les cas l'utilisation d'un multidétecteur permet de gagner énormément de temps (un facteur 10 environ) car on peut alors mesurer une plus grande partie du spectre à chaque acquisition. C'est particulièrement vrai en temps de vol où tout le spectre est mesuré en une fois. toutefois ceci à un prix assez élevé. L'intensité est beaucoup plus faible et mal répartie mais également la surface couverte de l'espace Qx-Qy est d'une forme peu pratique.

De plus, si ces mesures ont données des résultats qualitatifs raisonnables, seules des expériences sur des réseaux gravés ont données des résultats quantitatifs ${ }^{11}$ (voir ci-dessous). Ceci provient essentiellement du manque de flux qui impose de travailler au voisinage du plateau de réflexion totale.
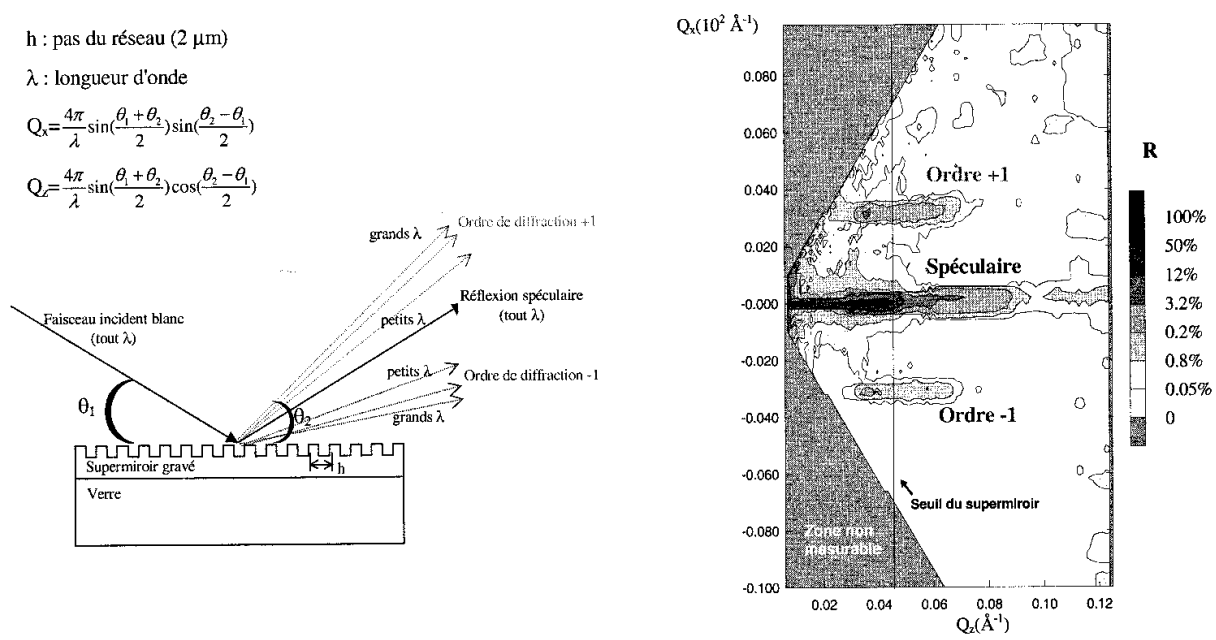

Figure 12 : Schéma du dispositif, et coefficient de réflexion mesuré (R) 


\section{$\underline{\text { Références }}$}

1 T.P. Russell Materials science reports, 5, 171-271, (1990)

2 . E. Fermi, Nuclear Physics, University of Chicago Press, 1949

3 . V.F. Sears, Neutron News, 3, 3, pp.26-37, 1992

4 . J. Penfold, R.K. Thomas, J. Phys. Condens. Matter, 2, pp.1369-1412, 1990 (voir p.1373)

5 . B. Farnoux, Neutron Scattering in the 'nineties, Conf. Proc. IAEA in Jülich, 14-18 janvier 1985, pp.205-209, Vienne, 1985

6 . X.D. Sun, Thèse de l'Université Paris Sud, Orsay, 1988.

${ }^{7}$ L.T. Lee, B. Jean, A. Menelle, Langmuir, 15, 3267-3272, (1999)

${ }^{8}$ C. Marzolin, Thèse de Doctorat en Physique, Paris VI, 15 Décembre 1995

${ }^{9}$ S.T. Milner et al., Macromolecules, 21, 2610, (1988)

${ }^{10}$ E.B. Zhulina et al., Macromolecules, 24, 4679-4690, (1991)

${ }^{11}$ F. Ott, Thèse de l'Université Paris Sud, Orsay, 1999 